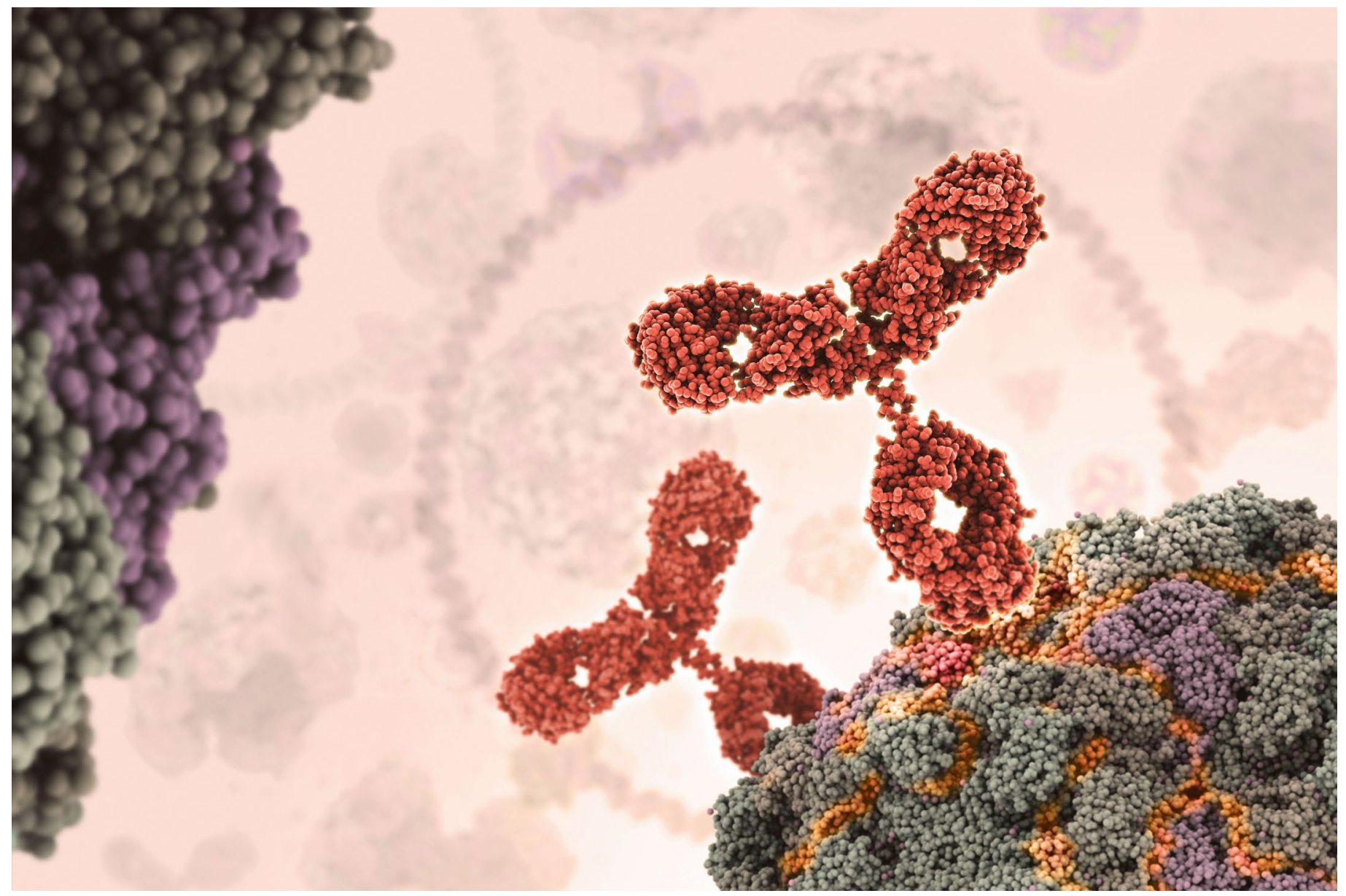

\title{
Refreshing the biologic pipeline 2020
}

In the absence of face-to-face meetings, FDA and industry implemented regulatory workarounds to maintain drug and biologics approvals. These could be here to stay.

\section{John Hodgson}

OVID-19 might have been expected to severely impair drug approvals in 2020. In the event, however, industry and regulators delivered a small miracle. They found workarounds and surrogate methods of engagement. Starting in January 2020, when the outbreak veered westward, the number of face-to face meetings declined rapidly; by March, they were replaced by Webex and Teams. (Secure Zoom meeting are to be added this year.) And remarkably, by 31 December, the US Food and Drug Administration (FDA) had approved nearly as many drugs and biologics in 2020 as it had in 2019 (itself the second highest year since 1996) - a small miracle in itself (Fig. 1 and Table 1).

To the usual crop of rare disease and genetic-niche cancer treatments, 2020 also added a chimeric antigen receptor (CAR)-T cell therapy with a cleaner manufacturing process and the first approved blockbuster indication for a small-interfering RNA (siRNA) - the European Medicines Agency's (EMA) registration of the RNA interference (RNAi) therapy Leqvio (inclisiran) for cardiovascular disease. Not to mention the rapid approvals and Emergency Use Authorizations (Box 1) for drugs against COVID-19.
"COVID-19 confronted us with the need to better triage sponsors' questions," says Peter Marks, the director of the Center for Biologics Evaluation and Research (CBER) at the FDA. "That was perhaps the single biggest takeaway from the pandemic related to product applications." Marks says that it became very apparent with some COVID19-related files that resolving a single issue can help a sponsor enormously and accelerate the development cycle. Before COVID-19, it was conceivable that a small company would have to wait 75 days - the standard schedule for a type $\mathrm{C}$ meeting even for relatively minor advice on whether one aspect of a manufacturing process or 


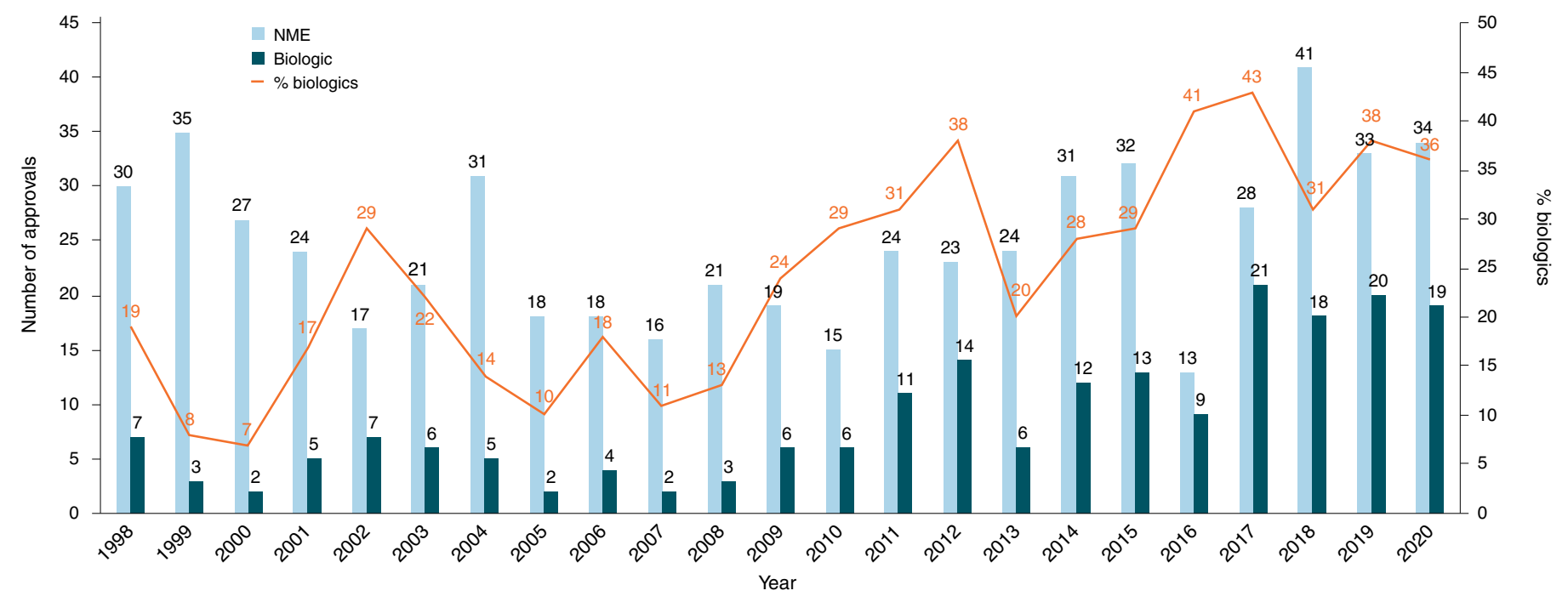

Fig. 1 | FDA approvals 2020. The regulatory agency seemingly did not miss a beat in 2020 , despite the pandemic.

control was better than another, or whether an animal model was appropriate. "It's a long time to wait," says Marks. "Going forward, we would like to find ways of increasing interactions so that sponsors' issues don't fester."

Another regulatory adjustment that accelerated the assessments of COVID-19 products was that clinical and manufacturing aspects were examined fully in parallel. Looking forward to the post-pandemic period, Marks says that, particularly in the area of vaccines and gene therapies, there is the potential "to significantly shorten the cycle times" both through advancing manufacturing technologies and through advisory processes on non-clinical and clinical programs characterized by an increased number of informal or less formal interactions. Furthermore, there was clear evidence during 2020 of two new and highly desirable directions in the regulation of drugs and biologics: moves toward patient-centered development and the internationalization of regulation.

\section{Going global}

One global regulatory initiative is Project Orbis, an FDA-led scheme under which a select group of national regulatory agencies are collaborating in assessing drug applications in oncology. A single regulatory submission to the FDA will be assessed collectively by experts from various agencies, leading, if the data are acceptable, to multiple approvals. However, control of the timing and precise nature of approvals may vary. Following a joint assessment, separate national agencies may impose different labeling and indication requirements for their territories. Project Orbis, in effect, is a corridor of assessment that opens multiple doors of similar and near-simultaneous approvals, but it does not enforce a uniform regulatory position.

The Project Orbis process began in September 2019 as the FDA, with its Australian and Canadian counterparts, approved a combination of two marketed drugs, in advanced endometrial carcinoma: the small-molecule multi-tyrosine-kinase inhibitor Lenvima (lenvatinib) from Eisai (Tokyo) and Merck's Keytruda (pembrolizumab), a humanized IgG4 monoclonal antibody (mAb) against programmed cell death receptor 1 (PD1). In 2020, regulatory bodies from Singapore (its Health Sciences Authority) and Switzerland (Swissmedic) joined the Project Orbis approval of its first new drug, Tukysa (tucatinib), a small-molecule inhibitor of HER2 developed by Seagen. Tukysa was approved in combination with Herceptin (trastuzumab) and Xeloda (capecitabine) for unresectable or metastatic HER2-positive breast cancer. The US label for Tukysa includes its use in patients with brain metastases, a growing indication that stems at least partly from the combination of prolonged survival due to directed antibody therapies (like Herceptin) and the inability of such antibodies to cross the blood-brain barrier.

Tukysa's approval was rapid, taking just 119 days from submission to approval, a record for new molecular entities under the FDA's Real-Time Oncology Review process that former FDA commissioner Scott Gottlieb introduced in 2018. The rapid process applies only to candidate drugs that show substantial top-line improvements over current therapy as demonstrated in cleanly designed trials. The FDA may now be considering expansion of the real-time review program to indications outside oncology.

The Brazilian Health Regulatory Agency (ANVISA) was also involved in some Project Orbis approvals during 2020. In October, the UK Medicines and Healthcare Products Regulatory Agency, faced with Brexit-related distancing from the EMA, decided to join not only Project Orbis but also the Access Consortium. Originally named for its regulatory membership from Australia, Canada, Switzerland and Singapore, the Access Consortium undertakes joint assessments of drugs beyond those in oncology, as well as of medical devices.

Two other new drugs were approved under Project Orbis in 2020: Zepzelca (lurbinectedin) from PharmaMar (Madrid) is a synthetic alkaloid derivative of an ecteinascidin from marine tunicates that promotes apoptosis by selectively inhibiting RNA polymerase II transcription in small-cell lung cancer; and Otsuka's Inqovi for myelodysplastic syndrome is an orally available fixed-dose combination of decitabine and cedazuridine, small-molecule inhibitors of cytosine DNA methyltransferase and cytidine deaminase, respectively.

For cell and gene therapy, however, moves towards international alignment were one of the first casualties of COVID-19. Regulators from the United States, Europe and Japan met face to face in Geneva in January 2020, but discussions may not resume for another six months or more "as soon as the COVID hurricane passes 
Table 1 | FDA biologics approvals in 2020

\begin{tabular}{|c|c|c|c|}
\hline Drug name & Generic name & Indication & Molecule type \\
\hline Blenrepa & Belantamab mafodotin-blmf & Multiple myeloma & $\begin{array}{l}\text { Humanized anti-BCMA IgG1 mAb conjugated to auristatin } \\
\text { F via a non-cleavable linker }\end{array}$ \\
\hline Danyelza & Naxitamab-gqgk & Neuroendocrine tumors & $\begin{array}{l}\text { Humanized IgG3 } 3 F 8 \text { mAb targeting ganglioside GD2 and } \\
\text { cluster of differentiation } 3\end{array}$ \\
\hline Darzalex Faspro & Daratumumab/hyaluronidase-fihj & Multiple myeloma & Fully human $\lg \mathrm{G} 1$ anti-CD38 mAb \\
\hline Ebanga & Ansuvimab-zykl & Ebola virus infection & $\begin{array}{l}\text { Fully human IgG1 mAb isolated from a survivor of the } \\
1995 \text { outbreak. }\end{array}$ \\
\hline Enspryng & Satralizumab-mwge & $\begin{array}{l}\text { Neuromyelitis optica (Devic's } \\
\text { syndrome) }\end{array}$ & Humanized IgG2 anti-interleukin- 6 receptor mAb \\
\hline Imcivree & Setmelanotide & Metabolic syndrome & $\begin{array}{l}\text { Small peptide agonist with specificity for melanocortin-4 } \\
\text { receptor }\end{array}$ \\
\hline Inmazeb & Atoltivimab/maftivimab/odesivimab-ebgn & Ebola virus infection & $\begin{array}{l}\text { Cocktail of three human IgG1 mAbs directed against } \\
\text { three Ebola epitopes }\end{array}$ \\
\hline Margenza & Margetuximab-cmkb & Breast cancer & $\begin{array}{l}\text { Fc optimized chimeric lgG1 anti-epidermal growth factor } \\
\text { receptor (EGFR) } \mathrm{mAb}\end{array}$ \\
\hline Monjuvia $^{a}$ & Tafasitamab-cxix & Diffuse large B-cell lymphoma & Fc optimized humanized IgG1 anti-CD19 mAb \\
\hline Phesgo & Trastuzumab/pertuzumab & Breast cancer & $\begin{array}{l}\text { Fixed dose combination of Herceptin and Perjeta } \\
\text { (pertuzumab), two anti-EGFR humanized lgG1 mAbs }\end{array}$ \\
\hline Sarclisa & Isatuximab-irfc & Multiple myeloma & Humanized IgG1 mAb against CD38 \\
\hline Sogroya & Somapacitan-beco & Short stature & $\begin{array}{l}\text { Recombinant human growth hormone analog with a single } \\
\text { substitution in the backbone (L101C) to which an albumin } \\
\text { is attached via a hydrophilic spacer }\end{array}$ \\
\hline Tecartus $^{a}$ & Brexucabtagene autoleucel & Mantle cell lymphoma & CD19-directed genetically modified autologous CAR-T cell \\
\hline Tepezza & Teprotumumab-trbw & Thyroid eye disease & IgG1 mAb that targets insulin-like growth factor 1 receptor \\
\hline Trodelvy ${ }^{a}$ & Sacituzumab govitecan-hziy & Breast cancer & $\begin{array}{l}\text { Humanized IgG1к monoclonal mAb conjugated with } \\
\text { SN-38, a topoisomerase inhibitor, using hydrolysable } \\
\text { linker CL2A }\end{array}$ \\
\hline Uplizna & Inebilizumab-cdon & Neuromyelitis optica & Humanized lgG4 anti-CD19 mAb \\
\hline Vyepti & Eptinezumab-jjmr & Migraine and other headaches & Humanized lgG1 anti-CGRP mAb \\
\hline \multicolumn{4}{|c|}{ Notable new molecular entities } \\
\hline Oxlumo & Lumasiran & Hyperoxaluria & siRNA oligonucleotide \\
\hline Viltepso & Viltolarsen & Duchenne muscular dystrophy & Phosphorodiamidate morpholino ASO \\
\hline
\end{tabular}

${ }^{a}$ Accelerated approval. mAb, monoclonal antibody; ASO, antisense oligonucleotide.

over," says Peter Marks. Regulatory practice evolves with exposure to sponsors' projects, and the relative newness and rarity of gene and cell therapy projects may mean that the evolution of regulations in different places is divergent. But there is a strong desire for alignment among the FDA, EMA and Japan's Pharmaceuticals and Medical Devices Agency, says Peter Marks. "For small patient populations, having divergent regulatory approaches is anti-innovation .... We would start with trying to get some of the technical requirements aligned, so that sponsors could use same dossier anywhere to support the same product."

\section{Going patient-centric}

Convenience is a watchword in drug approvals, both for patients and for the healthcare system. Inqovi is one example; the oral availability of the combination avoids the need for parenteral administration of decitabine. Several others joined it in 2020.

The European approval of Novartis's Leqvio (inclisiran) as the first siRNA drug for elevated low-density lipoprotein cholesterol (LDL-C) could mark one of the most fundamental shifts in the pharmaceutical market for decades. Thus far, siRNA drugs have been prominent as rare disease therapies, as the FDA approvals of Alnylam's second and third products demonstrate: Givlaari (givosiran), which targets 5-aminolevulinate synthase 1 inhibitor mRNA, was approved for acute hepatic porphyria in November 2019, and Oxlumo (lumasiran), which targets hydroxyacid oxidase 1 modulator mRNA, was approved for primary hyperoxaluria type 1 in November 2020. But Leqvio might take siRNA into a new dimension.

Targeted at the proprotein convertase subtilisin kexin 9 (PCSK9) transcript, Leqvio competes in the cholesterol-lowering market with anti-PCSK9 mAbs, such as Sanofi's Praluent (alirocumab, a human IgG1 $\mathrm{mAb}$ ) and Amgen's Repatha (evolucumab, a human IgG2 mAb) - drugs that were launched with a fanfare but have performed disappointingly in the market. However, even if Leqvio fails to become the blockbuster drug that Novartis hopes for and investors demand, 2021 will mark the year that siRNA as a technology joined mAbs in making the transition from orphan niche to pharma mainstream (Fig. 2).

Another move toward patient convenience came with the August approval for a once-weekly self-injection human 


\section{Box 1 | Lessons from Emergency Use Authorizations}

The FDA has issued many Emergency Use Authorizations (EUAs) during the pandemic, mostly for in vitro diagnostics and personal protective equipment, but also for a few therapeutics and vaccines (see table). EUAs are not approvals. They remain in effect only for the duration of the emergency and the reviews are less rigorous. Whereas regular FDA approvals will refer to "substantial evidence" of safety and efficacy, EUAs are issued on the basis of reasonable belief that products may be safe and effective. Although some lessons learned by regulators and drug developers may only apply to pandemic responses, other may be pointers for the future of drug development.

Lesson 1. The regulatory process flexes according to prevailing pressures (a lesson that actually applies to both EUAs and full approvals). It turns out, for instance, that face-to-face meetings with regulators are less important than the opportunity to exchange information on a regular basis, as long as security challenges are met. The way regulators adjusted during the COVID-19 pandemic is a particular reminder for executives in smaller companies that the authorities are best regarded as enablers rather than gatekeepers. For COVID- 19-related products, at least, regulators responded to the need for speed, considering regulatory steps in parallel rather than sequentially.

Lesson 2. Not every EUA will be given full approval, even when backed by high authority. US President Donald Trump's forceful espousal of the antimalarial generic hydroxychloroquine was one factor that led to its EUA at the end of March. Insufficient evidence of efficacy against SARS-CoV-2 infections led to revocation less than three months later. This is a clear reminder that although regulatory outcomes may combine elements of science, patient need and political context, evidence ultimately triumphs.

Lesson 3. Human plasma is a good starting point for anti-infective drug development programs. Passive immunization has been known to be an effective fix for infectious diseases since the beginning of the twentieth century, and so it proved with SARS-CoV-2. Three EUAs have been issued for passive immunization: the collection and use of convalescent plasma; Eli Lilly's single monoclonal antibody bamlanivimab; and Regeneron Pharmaceuticals' two-antibody cocktail of casirivimab and imdevimab. In October, full FDA approval was granted to another passive immunization therapy, Regeneron's Inmazeb, a three-monoclonal cocktail for the treatment of Zaire ebolavirus.

Lesson 4. Most small molecules are blunt tools for combatting a highly infectious viral respiratory pathogen, but in the absence of alternatives, ballpark activity can be enough. Gilead Science's Veklury (remdesivir) was not designed for SARS-CoV-2. It is an inhibitor of viral RNA-dependent RNA polymerase with broad in vitro antiviral action against a range of animal and human pathogens from many virus families. It got a US EUA on the basis that it appeared to shorten recovery times with SARS-Cov-2 if used early enough. Similarly, Lilly's Olumiant (baricitinib), a small-molecule inhibitor of Janus kinase (JAK) originally approved for rheumatoid arthritis, found a role in damping the cytokine storms that claimed many lives in COVID-19's first wave.

Lesson 5. For EUAs, the needs of the many outweigh those of the few. Innovation serves everybody.

\section{FDA Emergency Use Authorizations for combatting COVID-19}

\begin{tabular}{|c|c|c|c|}
\hline Intervention & Company & EUA date & Comments \\
\hline Chloroquine, hydroxychloroquine & - & 28 March & EUA revoked 15 June \\
\hline Veklury (remdesivir) & Gilead Sciences & 30 April & Antiviral, full approval 22 October \\
\hline COVID-19 convalescent plasma & - & 23 August & Passive immunotherapy \\
\hline Bamlanivimab & Eli Lilly & 9 November & Passive immunotherapy \\
\hline Olumiant (baricitinib) with remdesivir & Eli Lilly and Gilead Sciences & 19 November & Immune storm reduction, antiviral \\
\hline Casirivimab and imdevimab & Regeneron & 20 November & Passive immunotherapy \\
\hline COVID-19 vaccine & Pfizer and BioNTech & 11 December & mRNA vaccine \\
\hline COVID-19 vaccine & Moderna Therapeutics & 17 December & mRNA vaccine \\
\hline
\end{tabular}

EUA, emergency use authorization. Source: US FDA, https://www.fda.gov/emergency-preparedness-and-response/mcm-legal-regulatory-and-policy-framework/emergency-use-authorization\#covidtherap eutics

growth hormone (hGH) formulation, Novo Nordisk's Sogroya (somapacitan-beco), coming a mere 35 years after the approval of Genentech's recombinant human growth hormone, Protropin, which requires daily injections. Novo borrowed the technical key to Sogroya from the development of insulin detemir, the company's long-acting insulin analog: substituting cysteine for leucine at position 101 of hGH yields a molecule to which albumin can attach, extending the circulatory half-life from around 3-4 hours to 2-3 days.

A number of albumin-linked protein fusion drugs have been produced over the past decade, but not all have made the grade. The FDA approved CSL Behring's Idelvion (albutrepenonacog alfa), a recombinant albumin-human coagulation factor IX, in 2016. But both Teva Pharmaceuticals (Petah
Tikva, Israel) and GlaxoSmithKline have halted the development of their albuminated granulocyte colony stimulating factor (GCSF) products (Egranli (balugrastim) and Albugranin, respectively). Merrimack Pharmaceuticals' HER2-HER3-HSA (human serum albumin) bispecific fusion product floundered as the company ceased operational business. Having acquired Principia Pharmaceutical in 2000 for 


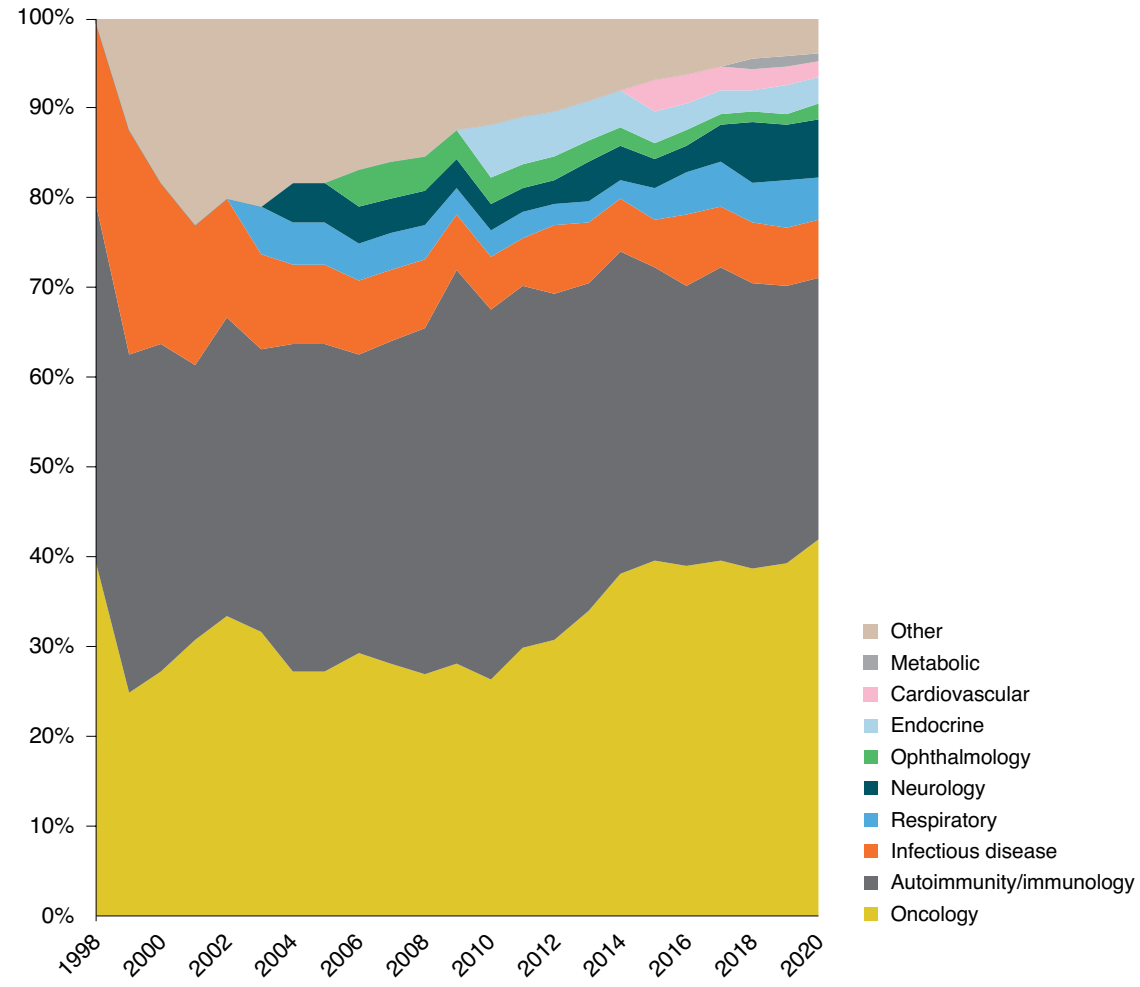

Fig. 2 | Monoclonal antibody approvals over time by disease group. Oncology and immunity and autoimmunity predominate, but other disease groups are slowly getting traction.

its albumin fusion technology, Human Genome Sciences developed Albuferon (albinterferon-alfa-2b) as a competitor to Roche's PEGylated $\alpha$-interferon Pegasys (peginterferon alfa-2a), as well as a long-lasting hGH, Albutropin. The half-lives of both were extended substantially. However, Human Genome Sciences stopped development of Albuferon-alfa in 2010 after an FDA ruling that it was no more effective than Pegasys. Albutropin is still in development at Teva.

Other new approvals will allow patients to avoid hospital-based treatment, a particularly desirable quality in the midst of COVID-19. Adding recombinant human hyaluronidase from Halozyme Therapeutics to a fixed-dose combination of Hoffmann-La Roche (Basel, Switzerland)/ Genentech humanized IgG1 anti-HER2 mAbs trastuzumab and pertuzumab led to the development of Phesgo (pertuzumab/ trastuzumab/hyaluronidase-zzfx). Because Phesgo is administered as a subcutaneous injection, its approval last June means that patients with HER2-positive breast cancers can now be treated outside infusion centers.

Safety considerations during COVID19 may have provided a more rapid route to market share for a second Roche product, Evrysdi (risdiplam). This drug was approved in August for treating spinal muscular atrophy (SMA) and is the first small-molecule exon-skipping drug, a selective splice modulator of survival of motor neuron 2 (SMN2) mRNA originally developed at PTC Therapeutics. Evrysdi is the third drug approved for SMA, and like Biogen's Spinraza (nusinersen), a 2'-O-2-methoxyethyl phosphorothioate antisense oligonucleotide developed originally by Ionis and approved in 2016, and Novartis's gene therapy Zolgensma (onasemnogene abeparvovec), a recombinant adeno-associated virus serotype 9 vector containing an SMN2 transgene under the control of a cytomegalovirus enhancer/chicken- $\beta$-actin hybrid promoter, approved in 2019, it works by increasing the amounts of full-length SMN2 mRNA and functional protein. Unlike Spinraza and Zolgensma, which are administered intrathecally and intravenously, respectively, and which both require hospitalization, Evrysdi comes as a strawberry-flavored drink formulated for infants that can be given at home. It is too early to say definitively how COVID-19 and the launch of Evrysdi altered the SMA market, but its noteworthy that sales of
Spinraza in the United States (but not in the rest of the world) were down $9 \%$ in the second quarter of 2020 and down 23\% in the third versus the corresponding quarters in 2019.

\section{Rare disease, common approval}

Regulatory systems around the world now strongly incentivize drug development for rare diseases. However, although orphan exclusivities can offer financial incentives for drug developers, low patient numbers pose their own conundrum - namely, whether it is ethical or practical to recruit patients with severe inherited disease without recourse to other treatments into a placebo arm of a trial.

The FDA and other regulators have shown sympathy for this position, allowing the control arms of clinical studies to be based on historical data on disease progression. One of the trials underpinning Evrysdi's approval examined how the drug slowed the loss of babies' abilities to sit or breathe unaided, compared with the course of untreated SMA.

At the far end of the rare disease spectrum is Hutchinson-Gilford progeria syndrome, a form of premature aging that affects only 1 in 18 million people. Symptoms show up in the first few months of life, and affected individuals die by their early teens, often from a stroke or a heart attack. In November, the FDA approved the first treatment, Eiger BioPharmaceuticals' Zokinvy (lonafarnib), an oral small-molecule farnesyltransferase inhibitor, on the basis of data that showed the drug extended the lifespan of treated patients by an average of 2.5 years compared with untreated ones.

Neurofibromatosis type 1 (NF1) affects around 1 in 3,000 people. Yet the approval in April of Cambridge, UK, AstraZeneca's Koselugo (selumetinib) for treating plexiform neurofibromas in NF1 also drew upon natural history data. AstraZeneca's data showed that the median reduction in the volume of painful, disfiguring tumors in nerve sheaths was $28 \%$ in children treated with selumetinib ${ }^{1}$ compared with a volume increase of at least $20 \%$ in age-matched controls from the US National Cancer Institute's natural history study of NF1.

One of the effects of orphan drugs legislation is that multiple drugs are approved for the same rare indication within a short period of time. Neuromyelitis optica spectrum disorder (NMOSD) is an autoimmune disease that affects the optic nerves and spinal cord in around 1-2 per 100,000 white people and around 5-10 per 100,000 Black people worldwide. It had no approved treatments before 2019. 
Now it has three, all $\mathrm{mAb}$ therapies. The first was Alexion's Soliris (eculizumab), a humanized monoclonal IgG2/4 $\mathrm{mAb}$ against complement protein $\mathrm{C} 5$ approved for NMOSD in 2019. The second arrived in June 2020 as the AstraZeneca autoimmunity and inflammation spin-off Viela Bio brought its first product to market: Uplizna (inebilizumab-cdon), a CD19-directed humanized afucosylated IgG1 mAb. The third, Enspryng (satralizumab-mwge), a humanized IgG2 mAb against the human interleukin- 6 receptor developed by Hoffmann-La Roche, followed swiftly, with FDA approval in August. Competition between the three products in this indication is likely to be intense, with only an estimated 4,000-8,000 people with NMOSD in the United States. Other products might join the throng soon: Alexion's longer acting (once every eight weeks) Soliris follow-up Ultomiris (ravulizumab-cwvz),

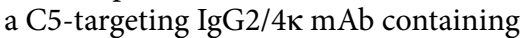
an $\mathrm{Fc}$ engineered for reduced neonatal Fc receptor binding, is in a phase 3 study (NCT04201262).

Similar product competition is seen in gene therapy for rare diseases, says Difei Yang, a senior biotech analyst at Mizuho Securities USA. She estimates, for example, that Duchenne muscular dystrophy (DMD) gene therapies from both Sarepta Therapeutics and Pfizer are likely be ready for Biologics License Application (BLA) submission within 6-12 months of each other. This alignment erodes any first-to-market advantage, something that is particularly relevant in the one-shot world of gene therapy. "Competition eventually leads to better and more choices for patients and physicians," says Yang, "And can be expected to put a lid on the high price tag of novel therapies."

Viltepso (viltolarsen) became the third approved exon-skipping drug for DMD when Japan's Ministry of Health, Labour and Welfare gave it the nod in March, nearly five months in advance of FDA's approval in August. Viltepso is a phosphorodiamidate morpholino antisense oligonucleotide that binds exon 53 of dystrophin pre-mRNA. It was developed by Nippon Shinyaku (Kyoto, Japan) and covers the same patient subgroup as Vyondys 53 (golodirsen) from Sarepta: the $8 \%$ of patients with confirmed dystrophin mutations susceptible to exon 53 skipping.

All four approvals so far in this class (including that for Viltepso) have accepted increased dystrophin production as a surrogate marker for efficacy but have required sponsors to demonstrate clinical benefit in postmarketing studies. Viltepso raises dystrophin levels to a greater extent (around five times more) than Vyondys 53.
A reckoning for exon-skipping drugs in DMD may be approaching. It is four years since the approval of Sarepta's Exondys 51 (eteplirsen) in 2016, and the company has a third compound, casimersen, due before the FDA in February.

As yet, none of these data have been published, but it all may become moot in the light of potential progress in DMD gene therapies and also next-generation exon skippers. Paul Matteis, a biotechnology analyst at Stifel, says that although the delivery of microdystrophin through gene therapy is "unlikely to be curative," it could still be "a huge win for patients and a big revenue generator." Matteis says that mechanistic synergies mean that the future of DMD could lie in combinations of microdystrophin gene therapy and next-generation exon skippers. Sarepta will have results of a placebo-controlled trial on DMD gene therapy in 2021.

\section{Optimizing adoptive cell therapy}

In July, the FDA approved Gilead Sciences' Tecartus (brexucabtagene autoleucel), autologous $\mathrm{T}$ cells engineered retrovirally ex vivo to express a CAR comprising a mouse anti-CD19 single-chain variable

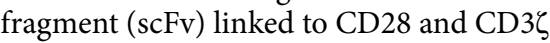
co-stimulatory domains, for the treatment of advanced mantle cell lymphoma. This was the second product stemming from Gilead's 2017 \$11.9 billion acquisition of Kite Pharma. Compared with that of Gilead's first approved CAR-T therapy, Yescarta (axicabtagene ciloleucel) for large B cell non-Hodgkin lymphoma, the manufacturing process for Tecartus has an extra $\mathrm{T}$ cell enrichment step that reduces the expansion of CD19-expressing tumor cells in the autologous cell preparation. In effect, Tecartus is a 'cleaner' CAR-T product.

Notwithstanding the greater product purity, the administration of Tecartus to patients remains burdensome, requiring treatment centers that are certified by the FDA and staffed by medical personnel trained to spot the treatment's severe side effects. At this point in development, adoptive cell therapy remains a last-ditch cancer treatment: patients in the trial on which the approval of Tecartus was based had all previously failed to respond not only to chemotherapy but also to treatment with a CD20 antibody (mAbs like rituximab, ocrelizumab, obinutuzumab, veltuzumab or ofatumumab) or a Bruton tyrosine kinase inhibitor (small molecules like ibrutinib, acalabrutinib or zanubrutinib).

\section{Getting under the skin}

Big pharma appreciates incremental technical improvements for its antibody products. This is because they not only improve the performance or convenience of a drug, but also build intellectual property protection for the originating company.

Halozyme's recombinant hyaluronidase platform, Enhanze, helps reposition biological drugs from specialist centers (such as hospitals or infusion centers) towards doctors' offices or home settings. Recombinant human hyaluronidase is an enzyme that acts as a permeabilizer, breaking down the subcutaneous polysaccharide found in the extracellular matrix of the skin and subcutaneous tissue and thus facilitating loading, dispersion and absorption of biologics. In effect, hyaluronidase allows biological drugs to be given through subcutaneous injection rather than through infusion.

Two more 'Enhanze-inside' $\mathrm{mAb}$ products were approved in 2020: Janssen Pharmaceutica's (Beerse, Belgium) Darzalex Faspro (a mixture of daratumumab, a

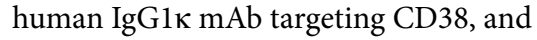
hyaluronidase-fihj) for the treatment of light-chain amyloidosis and Roche/ Genentech's Phesgo. These bring the number of approved products containing Halozyme's technology to six: the other three were Baxalta's HyQvia (hyaluronidase and a 10\% infusion of human polyclonal immune globulin) in 2014, Roche/Genentech's Rituxan Hycela (hyaluronidase and chimeric anti-CD-20 IgG1к mAb) in 2017, and Herceptin Hylecta (trastuzumab and hyaluronidase-oysk) in 2019. Halozyme's collaborative pipeline now includes Pfizer, Bristol Myers Squibb, Alexion and Eli Lilly, as well as Roche and Janssen.

\section{Antibody-drug conjugates loosen up} Another antibody adjunct that features prominently among 2020 drug approvals is Seagen's antibody-directed conjugate (ADC) technology. The first came in April with the approval of Immunomedics' Trodelvy (sacituzumab govitecan-hziy, a humanized IgG1 $\kappa \mathrm{mAb}$ targeting Trop-2 (trophoblast cell-surface antigen-2) conjugated to the topoisomerase inhibitor drug SN-38 via a hydrolysable CL2A linker) for third-line metastatic triple-negative breast cancer. The second Seagen ADC project to market in 2020 came in August with the approval of GlaxoSmithKline's Blenrep (belantamab mafodotin-blmf, an afucosylated humanized IgG1 mAb covalently linked to the microtubule inhibitor monomethyl auristatin $\mathrm{F}$ via a protease-resistant maleimidocaproyl linker) for multiple myeloma.

For Trodelvy, the path to market was far from smooth. The roots of the compound go back at least to 2004, when Immunomedics 
developed a humanized antibody, hRS7, against Trop-2 (epithelial glycoprotein-1), an antigen overexpressed on numerous tumor types. In 2017, the compound was to be the cornerstone of a major collaboration between Immunomedics and Seagen (at the time called Seattle Genetics). Seattle Genetics, which already had Adcetris (brentuximab vedotin, a chimeric IgG1 $\mathrm{mAb}$ targeting CD30 conjugated to the microtubule inhibitor monomethyl auristatin E via a protease-cleavable linker) on the market, was to assume responsibility for manufacturing development and taking the compound through the regulatory process. Key Immunomedics shareholders argued that the deal has been "rushed" and did not deliver value to shareholders. The complete response letter issued by FDA in January 2019 outlining concerns about aspects of Trodelvy's submission related to chemistry, manufacturing and control added further fuel to the fire. But ultimately, the partnership was vindicated - first by the drug's approval in April 2020 and then again, in September 2020, when Gilead made its $\$ 21$ billion offer for Immunomedics.

Trodelvy has been described by the company and its advisors as "a pipeline in a product" because it represents a different approach to ADCs. Early ADCs (like Adcetris) bound a single, highly toxic molecular payload tightly to an antibody. Tumor killing ensued when the complex of bound antibody and toxin was internalized and the linker proteolytically degraded. Trodelvy, by contrast, puts up to eight copies of a moderately toxic compound (SN-38) onto a single antibody. The linker chemistry can be tuned so that, in addition to internalization, the toxin is released locally under the physiological conditions of the tumor ${ }^{2}$.

\section{Fc engineering to the fore}

The accelerated approval of Monjuvi (tafasitamab-cxix, an anti-CD19 humanized IgG1/2 mAb containing a hybrid Fc domain with two amino acid substitutions to modify the Fc-mediated functions) at the end of July made it the second approved product for MorphoSys (Planegg, Germany) and the second approved therapeutic to use Xencor's XmAb Fc-domain-engineering technology. Xencor earned a \$25 million regulatory milestone. The first $\mathrm{XmAb} \mathrm{mAb}$ on the market was Ultomiris (see "Rare disease, common approval" above). For Monjuvi, $\mathrm{XmAb}$ engineering increased the level of natural killer (NK) cell-mediated antibody-directed cellular cytotoxicity (ADCC) in vitro ${ }^{3}$. Monjuvi's initial indication is confined to a narrow patient subgroup (as a second-line treatment in relapsed or refractory diffuse large B cell lymphoma in patients who are not eligible for an autologous stem cell transplant) and has to be used in combination with lenalidomide (Celgene's Revlimid). However, MorphoSys and US partner Incyte hope to expand its use to a range of lymphoma indications.

A second Fc-engineered $\mathrm{mAb}$ was approved on 16 December: MacroGenics' Margenza (margetuximab-cmkb), a

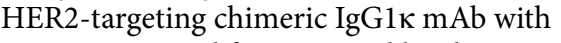
an $\mathrm{Fc}$ engineered for increased binding of activating Fc $\gamma$ receptor 3A (CD16A) and decreased binding to inhibitory Fcy receptor $2 \mathrm{~B}(\mathrm{CD} 32 \mathrm{~B})$. These changes lead to greater in vitro ADCC and NK cell activation. Margenza, MacroGenics' first approved product, outperformed Herceptin in a head-to-head trial, particularly in patients with low-affinity CD16A genotypes, the group for whom trastuzumab is less effective.

\section{Genetically targeted drugs continue march}

So called precision cancer drugs are one of biotechnology's stocks-in-trade. Six were approved in 2020 (Table 2). The use of a genetic marker enables clinical development costs to be mitigated by targeting a development program to highly selected small numbers of patients. The flagship product is Novartis's Gleevec (imatinib), a small-molecule drug approved in 2001 for chronic myelogenous leukemia (CML) with a chromosome 9 and 22 fusion (Philadelphia chromosome). Gleevec transformed five-year survival rates from $30 \%$ to around 89\%.

Gleevec pioneers Brian Druker of Oregon Health \& Science University and Nick Lydon, then of Ciba-Geigy Pharmaceuticals, went on to found Blueprint Medicines in Boston in 2008. Twelve years later, Blueprint's first two product approvals arrived: Ayvakit (avapritinib), a small-molecule multiple tyrosine kinase inhibitor for patients with a platelet-derived growth factor receptor A D842V mutation in exon 18, in January 2020, and Gavreto (pralsetinib), a small-molecule multiple tyrosine kinase inhibitor for patients with a RET gene fusion (CCDC6-RET) and V804L, V804M and M918T mutations, in September.

Most of these approvals will not have an impact anything like that of Gleevec. Whereas the cancer cells in nearly all cases of CML carry the Philadelphia chromosome and are likely susceptible to Gleevec, specific genetic rearrangements account for only a fraction of other cancers. For instance, the fibroblast growth factor receptor 2 (FGFR2) fusion target for Incyte's Pemazyre (pemigatinib), a small-molecule kinase inhibitor targeting FGFR1, FGFR2 and

Table 2 | Gene-targeted drugs approved in 2020

\begin{tabular}{|c|c|c|c|c|}
\hline Drug & Generic name & Indication & Developer & Date approved \\
\hline Ayvakit & Avapritinib & GIST harboring a PDGFRA exon 18 mutation & Blueprint Medicines & $\begin{array}{l}9 \text { January (FDA), } \\
28 \text { July (EMA) }\end{array}$ \\
\hline Pemazyre & Pemigatinib & $\begin{array}{l}\text { Cholangiocarcinoma harboring an in-frame FGFR2 gene fusion (with a } \\
\text { breakpoint within intron17/exon 18) or non-fusion rearrangement }\end{array}$ & Incyte & 17 April \\
\hline Tabrecta & Capmatinib & NSCLC harboring MET exon 14 mutations & Novartis & 6 May \\
\hline Retevmo & Selpercatinib & $\begin{array}{l}\text { NSCLC harboring RET mutations in the extracellular cysteine-rich } \\
\text { region (M918T, V804M, V804L or others) }\end{array}$ & Loxo Oncology/Eli Lilly & 8 May \\
\hline Tazverik & Tazemetostat & $\begin{array}{l}\text { Relapsed or refractory follicular lymphoma with mutations in EZH2 } \\
\text { leading to loss of integrase interactor } 1\end{array}$ & Epizyme/Royalty Pharma & 18 June \\
\hline Gavreto & Pralsetinib & $\begin{array}{l}\text { NSCLC harboring RET gene fusion (CCDC6-RET) or V804L, V804M } \\
\text { and M918T mutations }\end{array}$ & Blueprint Medicines & 4 September \\
\hline
\end{tabular}

GIST, gastrointestinal stromal tumor; FGFR2, fibroblast growth factor receptor 2; NSCLC, non-small-cell lung cancer; MET, mesenchymal-epithelial transition gene; RET, the RET proto-oncogene; PDGFRA platelet-derived growth factor receptor- $\alpha$; EZH2, enhancer of zeste homolog 2; CCDC6, coiled-coil domain containing 6. 
FGFR3 for patients with an in-frame FGFR2 gene fusion (with a breakpoint in intron17/ exon 18) or non-fusion rearrangement, is seen in just $9-14 \%$ of patients with cholangiocarcinoma. Similarly, although around $70 \%$ of non-small-cell lung cancers have a genomic mutation of some kind, only 3-4\% lead to skipping of $M E T$ exon 14 (METex14), the oncogenic driver against which Novartis's Tabrecta (capmatinib), a small-molecule inhibitor of MET kinase, is directed.

For Epizyme, the developer of Tazverik (tazemetostat), a small-molecule inhibitor of the methyltransferase EZH2, the limited patient population (the drug is approved only for patients with mutations leading to loss of integrase interactor 1) means that the company may struggle to pay back its investment in the compound. Epizyme has assigned the royalties due from Eisai on sales of Tazverik in Japan to Royalty Pharma and arranged a $\$ 70$ million line of credit with Pharmakon Advisors, a Royalty subsidiary, to fund the regulatory milestones it owes Eisai. US sales of Tazverik since its approval are below $\$ 5$ million per quarter.

For another 2020-approved precision drug, Retevmo (selpercatinib), a small-molecule multikinase inhibitor indicated for patients with RET mutations in the extracellular cysteine-rich region (M918T, V804M V804L and others), the business story is already well advanced. Retevmo was a prominent asset in Eli Lilly's early-2019 $\$ 8$ billion acquisition of Loxo Oncology. Another compound that fits this category is Blueprint's Gavreto (see above); in July 2020, Roche paid $\$ 675$ million up front plus a $\$ 100$ million equity investment for non-US, non-China co-development and co-commercialization rights on the basis of the results of the ARROW clinical trial.

The synergy between biotech firms and big pharma is likely to persist, says Mizuho's Yang. "Biotech brings strengths in technology innovation and an approach to clinical validation that brings products forward in the most valuable indications or disease settings. Pharma, on the other hand, has the wherewithal to navigate complex regulatory environments, invest in new manufacturing technologies, and work with payers/physicians/patients to optimally commercialize new therapies" (Box 2).

\section{Looking forward to 2021}

The inauguration of Joe Biden as president of the United States on 20 January 2021 is unlikely to impact drug approval policy, but it may affect pricing. MassBio's Director of Government Affairs, Susan Martin, says

\section{Box 2 | Business journeys}

Some of the 2020 roster of approved drugs traveled far from their origins (see Table). Between developer and their current owner, mAbs such as Sarclisa (isatuximab-irfc), a CD38-directed chimeric IgG1 mAb, and Vyepti (eptinezumab-jjmr), a humanized IgG1 mAb against calcitonin gene-related peptide (CGRP) ligand, crossed the Atlantic Ocean. Xeglyze (abametapir), a small-molecule metalloproteinase inhibitor for treating human head lice, is marketed by India's Dr Reddy's Laboratories, but started life as an infestation control agent in the Australian poultry company HatchTech.
Other drugs have travelled circuitously. Tepezza (teprotumumab-trbw), a fully human IgG1 mAb targeting insulin-like growth factor-1 receptor) was originally developed by Danish company Genmab, subsequently licensed to Roche, returned to Genmab, and developed further by River Vision (a company established solely to develop the asset) before finally being sold to Horizon Pharma in 2017.

But the award for the most time-consuming journey must go to Byfavo (remimazolam), a short-acting anesthetic $\gamma$-aminobutyric acid A $\left(\mathrm{GABA}_{\mathrm{A}}\right)$ receptor agonist that is indicated for procedural sedation in adults. This benzodiazepine started as a germ of an asset at GlaxoSmithKline before being bartered in 2003 to Cambridge, UK, startup $\mathrm{CeNeS}$ Pharmaceuticals in exchange for a $5 \%$ stake. In 2008, the drug was sold to Paion (Aachen, Germany) in an all-stock transaction valued at $\$ 21.7$ million. Four years ago, Dublin, Ireland-based Cosmo Pharmaceuticals in-licensed the US rights to remimazolam and joined Paion in efforts to progress the candidate to registration. In July 2020, Cosmo sold the license to Acacia Pharmaceuticals, which received FDA approval in October 2020.

\section{Acquired approvals}

\begin{tabular}{|c|c|c|c|c|}
\hline Product & Current owner & Previous developer & Mode of acquisition & Indication \\
\hline Sarclisa (isatuximab-irfc) & Sanofi & Immunogen & Licensed product & Third-line multiple myeloma \\
\hline Vyepti (eptinezumab-jjmr) & Lundbeck (Valby, Denmark) & Alder BioPharmaceuticals & $\begin{array}{l}\text { Bought Alder for } \\
\$ 1.95 \text { billion in } 2019\end{array}$ & Migraine prevention \\
\hline Tukysa (tucatinib) & Seagen & $\begin{array}{l}\text { Array BioPharma via Cascadian } \\
\text { Therapeutics }\end{array}$ & $\begin{array}{l}\text { Bought Cascadian for } \\
\$ 614 \text { million in } 2018\end{array}$ & $\begin{array}{l}\text { Advanced HER2-positive } \\
\text { breast cancer }\end{array}$ \\
\hline $\begin{array}{l}\text { Tepezza } \\
\text { (teprotumumab-trbw) }\end{array}$ & Horizon Therapeutics & $\begin{array}{l}\text { Genmab via River Vision and } \\
\text { Roche }\end{array}$ & $\begin{array}{l}\text { Bought River Vision for } \\
\$ 145 \text { million in } 2017\end{array}$ & Thyroid eye disease \\
\hline $\begin{array}{l}\text { QWO (Clostridium } \\
\text { histolyticum collagenase) }\end{array}$ & Endo & BioSpecifics Technologies & $\begin{array}{l}\text { Bought BioSpecifics for } \\
\$ 540 \text { million in } 2020\end{array}$ & Cellulite \\
\hline Zeposia (ozanimod) & Bristol Myers Squibb & Celgene & $\begin{array}{l}\text { Bought Celgene for } \\
\$ 74 \text { billion in } 2019\end{array}$ & Relapsing multiple sclerosis \\
\hline Byfavo (remimazolam) & Paion (Aachen, Germany) & $\begin{array}{l}\text { GlaxoSmithKline via CeNeS } \\
\text { Pharmaceuticals }\end{array}$ & $\begin{array}{l}\text { Bought CeNeS for } \\
\$ 22 \text { million in } 2008\end{array}$ & Procedural sedation \\
\hline Xeglyze (abametapir) & Dr. Reddy's Laboratories & HatchTech & Licensed product & Head lice infestation \\
\hline $\begin{array}{l}\text { Tecartus (brexucabtagene } \\
\text { autoleucel) }\end{array}$ & Gilead Science & Kite Pharma & $\begin{array}{l}\text { Bought Kite for } \\
\$ 11.9 \text { billion in } 2017\end{array}$ & $\begin{array}{l}\text { Advanced mantle cell } \\
\text { lymphoma }\end{array}$ \\
\hline
\end{tabular}


Biden's support for a form of international pricing index for Medicare drugs and policies that aim to keep drug prices in step with inflation makes the new president "worse on drug pricing reform for the life sciences industry than President Trump, but not by much."

Until the northern hemisphere winter season for respiratory diseases has passed, most of the public attention around the biotech and pharmaceutical industry sectors will remain focused on the progress of COVID-19-related therapeutics and vaccines. "COVID-19 has been agenda item 1, 2 and 3 for regulators everywhere," says CBER's Marks. However, in preparing for the next pandemic, Marks believes that, in addition to keeping abreast of biological innovations, it will also be important for industry and regulators to rise to the challenge of manufacturing innovations in huge quantities. "For antibodies, even for vaccines, could we see continuous or semicontinuous manufacturing rather than batch processes? We need very much to be working towards this in order to be better prepared for the next pandemic," he says.

Within investor and industry circles, key regulatory decisions scheduled for the first quarter of 2021 will help delineate the role of humanized mAbs in substantial indications, such as Alzheimer's disease, osteoarthritis and metabolic disease (Fig. 2).

In the last of these, increasing therapeutic options for cholesterol-lowering drugs, including the launch of the siRNA Leqvio, portend an increasingly competitive market. Regeneron's evinacumab, a fully human IgG1 mAb against angiopoietin-like 3 protein, is expected to go before the FDA in February 2021 and would become the first $\mathrm{mAb}$ to be used for homozygous familial hypercholesterolemia. Although homozygous familial hypercholesterolemia affects only around 1,300 people in the United States, Regeneron also has the antibody in advanced clinical trials in broader hypercholesterolemia indications.

In March, the FDA is expected to look at Pfizer and Eli Lilly's tanezumab, a humanized IgG2 heavy chain and $\kappa$-light chain $\mathrm{mAb}$, one of two antibodies targeted against nerve growth factor (NGF) in late-stage development (the other is Regeneron and Teva's fully human IgG1 $\mathrm{mAb}$ fasinumab). The issue with anti-NGF agents has been that some treated patients' osteoarthritic disease has worsened, perhaps because the agents' potent pain relief has allowed patients to be more active. Nevertheless, this observation has restricted the clinical programs for both tanezumab and fasinumab to the use of low-dose preparations. Two earlier trials of tanezumab, in osteoarthritis of the knee and hip, were halted in 2009 and 2010, respectively, because of worsening disease. Any approval of tanezumub is likely to be restricted to patients who are unable to use standard-of-care analgesics, such as non-steroidal anti-inflammatory drugs.

Also in March, the FDA is expected to pass judgment on Biogen's controversial strategy with the Alzheimer's drug aducanumab, a chimeric human IgG1 $\mathrm{mAb}$ targeting $\beta$-amyloid. The decision has much at stake, according to Stifel analyst Matteis. Beyond aducanumab potentially representing the first disease-modifying Alzheimer's drug, its success or failure will have a profound impact on Biogen's profitability. Matteis believes that the aducanumab decision will be an indicator of FDA flexibility in the post-Gottlieb era, both in Alzheimer's disease and in neurology generally.

No surprise, then, that before the FDA Advisory Committee for aducanumab last November, analysts at Canaccord Genuity called the antibody "biopharma's most closely-watched pipeline product" outside COVID. The evidence for the effectiveness of
Biogen's aducanumab has been equivocal at best. Indeed, Biogen's own view of the drug candidate's performance has flip-flopped. In March 2019, the company announced aducanumab did not meet its primary endpoints of slowing cognitive decline in the ENGAGE and EMERGE phase 3 clinical studies. But in October that year, it did an about-face, arguing that reanalyzed evidence from EMERGE indicated that high-dose aducanumab had slowed cognitive decline. Biogen leaked that the FDA had indicated to the company that it planned to act early on aducanumab, but the responses from the agency's Peripheral and Central Nervous System Drugs Advisory Committee on 6 November 2020 were largely, if not overwhelmingly, negative.

In the light of the negative advisory committee, an FDA approval of aducanumab would be "pretty controversial," says Matteis. "But investors might then view FDA as more accommodating, sending positive signals to other companies working in Alzheimer's, where a good deal of medical need will remain unmet." Across neurology, where clinical development is notoriously difficult, Matteis says that a positive decision in March for aducanumab might help reaffirm that drugs can get approved with some positive and some negative data. But historical precedents, at least from the past 15 years, are unpromising: "We've been unable to find a drug that's gotten approved after zero AdComm panelists voted in favor," says Matteis.

\section{John Hodgson}

Cambridge, UK.

Published online: 28 January 2021

https://doi.org/10.1038/s41587-021-00814-w

References

1. Gross, A. M. et al. N. Engl. J. Med. 382, 1430-1442 (2020).

2. Goldenberg, D. M. et al. Oncotarget 6, 22496-22512 (2015).

3. Kellner, C. et al. Leukemia 27, 1595-1598 (2013). 